\title{
OUTILS D'AIDE À LA TRADUCTION DE LA LÉGISLATION DE L'UE : PRATIQUES EUROPÉENNES ET CAS DU BCMS
}

Olivera Vušović, Université du Monténégro, oliverav@ucg.ac.me

10.31902/fll.29.2019.12

UDK 025.43 :061.1EU

UDK 81'272(4)

Résumé : Le présent article vise à aborder la question de mémoire à travers les outils de stockage de données linguistiques (bases terminologiques, corpus parallèles, thésaurus, glossaires et dictionnaires) déployés dans le contexte de la traduction de la législation de l'UE. D'un côté, nous présentons le panorama des outils utilisés au sein de l'UE, qui se distingue par un régime linguistique complexe et fort spécifique, comprenant actuellement 24 langues officielles. De l'autre côté, nous passons en revue les préparatifs et la mise en place de divers outils dans les pays de l'ancien serbo-croate, à savoir le cadre linguistique de leur adhésion à l'UE. Au sein des langues BCMS, il convient de tracer un parallèle entre la Croatie, pays membre de l'UE depuis 2013, disposant des mécanismes les plus déployés en la matière, d'une part, et le Monténégro, la Serbie et la Bosnie-Herzégovine, d'autre part.

Mots-clés : Union européenne, traduction, outils, bases de données, adhésion à l'UE, BCMS.

\section{Introduction}

« Sans les ponts jetés par la traduction, il aurait été impossible de concevoir une union entre les peuples de l'Europe » (Commission européenne 2009, 5).

Les efforts d'unification économique et politique des pays du continent européen s'intensifient après la Seconde guerre mondiale. La déclaration Schuman du 9 mai 1950 mène à la signature de Traité de Paris le 18 avril 1951 et à la création de la Communauté européenne du charbon et de l'acier (CECA), pour une durée de 50 ans, regroupant la France, la République fédérale d’Allemagne, I'Italie et les pays du Benelux (la Belgique, le Luxembourg et les PaysBas). C'était la première organisation supranationale à l'échelle européenne.

Les six pays cherchent bientôt à intensifier leur coopération en établissant un marché commun autorisant la libre circulation des personnes, marchandises et capitaux. Les traités de Rome du 25 mars 1957 instaurent la Communauté économique européenne (CEE) et la Communauté européenne de l'énergie atomique (CEEA ou Euratom). 
Une nouvelle étape de l'unification européenne est entamée par le Traité de Maastricht, signé le 7 févier 1992, mettant en place l'union politique et économique qui prend le nom de l'Union européenne.

L'une des caractéristiques essentielles de I'Union européenne qui la distingue de toute autre organisation supranationale à l'échelle mondiale est un nombre très élevé de langues officielles (pour l'instant, il est à 24). Les six pays qui formaient la Communauté européenne du charbon et de l'acier (CECA) étaient dès le début décidés à fixer le statut d'égalité de toutes les langues. L'intention de maintenir ce régime linguistique a perduré au fur et à mesure de la restructuration et des élargissements des Communautés européennes. " Le multilinguisme adopté dès le début apparaît donc comme une solution pragmatique : car en adoptant un nombre réduit de langues, la Communauté aurait ouvert la porte à de nouveaux conflits et se serait aliéné la sympathie des citoyens européens » (Commission européenne 2010, 10).

Le fait que toutes les versions linguistiques des actes du droit de l'UE sont également authentiques révèle « toute la dimension du travail de médiation interculturelle qu'effectue le traducteur » (Commission européenne 2009, 13). Au cours des cinq décennies d'existence de cette vaste union politique, juridique et culturelle, " la traduction a su s'adapter aux multiples défis en se restructurant et en se montrant toujours plus innovante " (Commission européenne 2010, 6).

Dans les lignes qui suivent, notre intention est, d'un côté, de rendre compte de la pratique actuelle dans les institutions de l'UE, tout en passant en revue le panorama des outils d'aide à la traduction déployés au sein de l'UE et de l'autre côté, nous comptons nous pencher sur les préparatifs et la mise en place de divers outils dans les pays de l'ancien serbo-croate, à savoir sur le cadre linguistique de leur adhésion à l'UE.

\section{Pratiques actuelles au sein de l'UE}

Selon De Vicente (2011), l'effectif des traducteurs dans l'ensemble des institutions de l'UE (sans compter les juristes-linguistes) prend la forme suivante :

\begin{tabular}{|l|c|}
\hline Institution & Effectif des traducteurs \\
\hline Commission européenne & 1750 \\
\hline Parlement européen & 760 \\
\hline Conseil & 650 \\
\hline Cour de justice & 620 \\
\hline Cour des comptes & 100 \\
\hline
\end{tabular}




\begin{tabular}{|l|c|}
\hline Institution & Effectif des traducteurs \\
\hline $\begin{array}{l}\text { Comité des régions et Comité économique } \\
\text { et social européen }\end{array}$ & 350 \\
\hline Banque centrale européenne & 70 \\
\hline
\end{tabular}

Tableau 1. Effectif des traducteurs dans l'ensemble des institutions de l'UE (De Vicente 2011)

Comme nous pouvons le voir, la Commission européenne se distingue par un effectif considérable. Son service de traduction, la Direction Générale de la Traduction (DGT), a de plein droit la réputation d'être l'un des plus grands services de traduction au monde. Sa production s'élève à « quelque 2 millions de pages par an » (Commission européenne 2013, 1). La problématique de la traduction n'est pas identique dans toutes les institutions européennes. La Commission, le Conseil et le Parlement travaillent en effet principalement en anglais, en allemand et en français. Leurs textes de travail sont en principe corédigés dans ces langues et ne sont traduits dans les autres langues officielles que dans un second temps, en amont de la procédure législative. [...] Ces institutions ont choisi de recruter des traducteurs non juristes ; elles leur ont néanmoins adjoint les services de quelques " juristes-réviseurs ", c'est-à-dire de juristes chargés d'assurer le respect de la légistique et la cohérence entre les différentes versions linguistiques (Pellerin Rugliano 258).

La Cour de justice de l'UE présente quelques particularités par rapport aux autres institutions. Depuis sa création, " la Cour délibère dans une seule et même langue, le français, et le projet d'arrêt adopté par les juges, au terme de leur délibéré, est écrit en français avant d'être traduit dans la langue de procédure dans laquelle il va être prononcé » (Commission européenne 2009, 13).

En ce qui concerne le personnel chargé de l'activité traduisante, Pellerin Rugliano, juriste-linguiste à la Cour de justice de l'Union européenne souligne que, depuis son instauration, cette institution " a préféré ne recruter que des juristes pour assurer ses traductions » (Pellerin Rugliano 259). Selon le règlement de procédure de la Cour :

\section{Article 42 Service linguistique de la Cour}

La Cour établit un service linguistique composé d'experts justifiant d'une culture juridique adéquate et d'une connaissance étendue de plusieurs langues officielles de l'Union.

Que ce principe implique-t-il en pratique ? " La Cour exige que l'impétrant ait achevé un cursus juridique complet, soit cinq ans d'études de droit réa- 
lisées dans la langue pour laquelle le concours est organisé » (Pellerin Rugliano 259). Le diplôme de traducteur ne suffit donc pas pour postuler. Les candidats sélectionnés :

Sont tenus de réaliser un stage d'aptitude de neuf mois, qui correspond à une période d'essai. Pendant ces neuf mois, chaque texte traduit par le fonctionnaire stagiaire fait l'objet d'une relecture critique et d'une correction par un juriste-réviseur, c'est-à-dire un juriste-linguiste plus expérimenté (Pellerin Rugliano 272).

\section{Outils d'aide à la traduction}

Aujourd'hui, les technologies de l'information facilitent le travail des traducteurs au sein des institutions européennes, au point que cette activité devient inconcevable sans elle. Le panorama de principales ressources d'aide à la traduction, déployées au sein de la Commission européenne, prend la forme suivante :

\begin{tabular}{|l|l|}
\hline Outil & Description \\
\hline Poetry & $\begin{array}{l}\text { Logiciel d'envoi électronique des demandes des autres } \\
\text { services de la Commission } \\
\text { à la DG Traduction }\end{array}$ \\
\hline Suivi & $\begin{array}{l}\text { Logiciel de gestion électronique des demandes de } \\
\text { traduction à l'intérieur } \\
\text { de la DG Traduction }\end{array}$ \\
\hline Euramis & Mémoire de traduction centrale de la DG Traduction \\
\hline TraDesk & $\begin{array}{l}\text { Interface de gestion des traductions et système d'accès } \\
\text { aux archives électroniques } \\
\text { où les traductions antérieures sont stockées }\end{array}$ \\
\hline DGTVista & Moteur de recherche et de visualisation de documents \\
\hline EUR-Lex & $\begin{array}{l}\text { Base de données en ligne du droit de l'Unioneuropéenne } \\
\text { (http://eur-lex.europa.eu) }\end{array}$ \\
\hline IATE & $\begin{array}{l}\text { Base terminologique de toutes les institutions de } \\
\text { l'Union européenne } \\
\text { (http://iate.europa.eu) }\end{array}$ \\
\hline
\end{tabular}

Tableau 2.

Aperçu des outils d'aide à la traduction (Commission européenne 2013,4) 
Les demandes de traduction sont transmises électroniquement à la DG Traduction à l'aide du logiciel Poetry. Un autre logiciel, Suivi, gère les demandes et " permet d'attribuer les traductions aux unités et aux traducteurs et de fournir les traductions aux services demandeurs » (Commission européenne 2013, 6). La mémoire de traduction centrale Euramis (European Advanced Multilingual Information System) assure un bon partage de données entre plusieurs institutions de l'UE, permet d'éviter le travail répétitif et contribue à une meilleure cohérence linguistique des documents traduits.

Actuellement, la mémoire centrale d'Euramis contient près de 645 millions de segments couvrant toutes les langues officielles de I'Union européenne. L'anglais et le français sont les langues sources les plus utilisées, puisque la quasi-totalité des documents produits par la Commission sont rédigés dans l'une de ces deux langues (Commission européenne 2013, 11).

Le cycle de la traduction à la Commission européenne est présenté de manière suivante : 


\section{LE CYCLE DE LA TRADUCTION À LA COMMISSION EUROPÉENNE (*)}

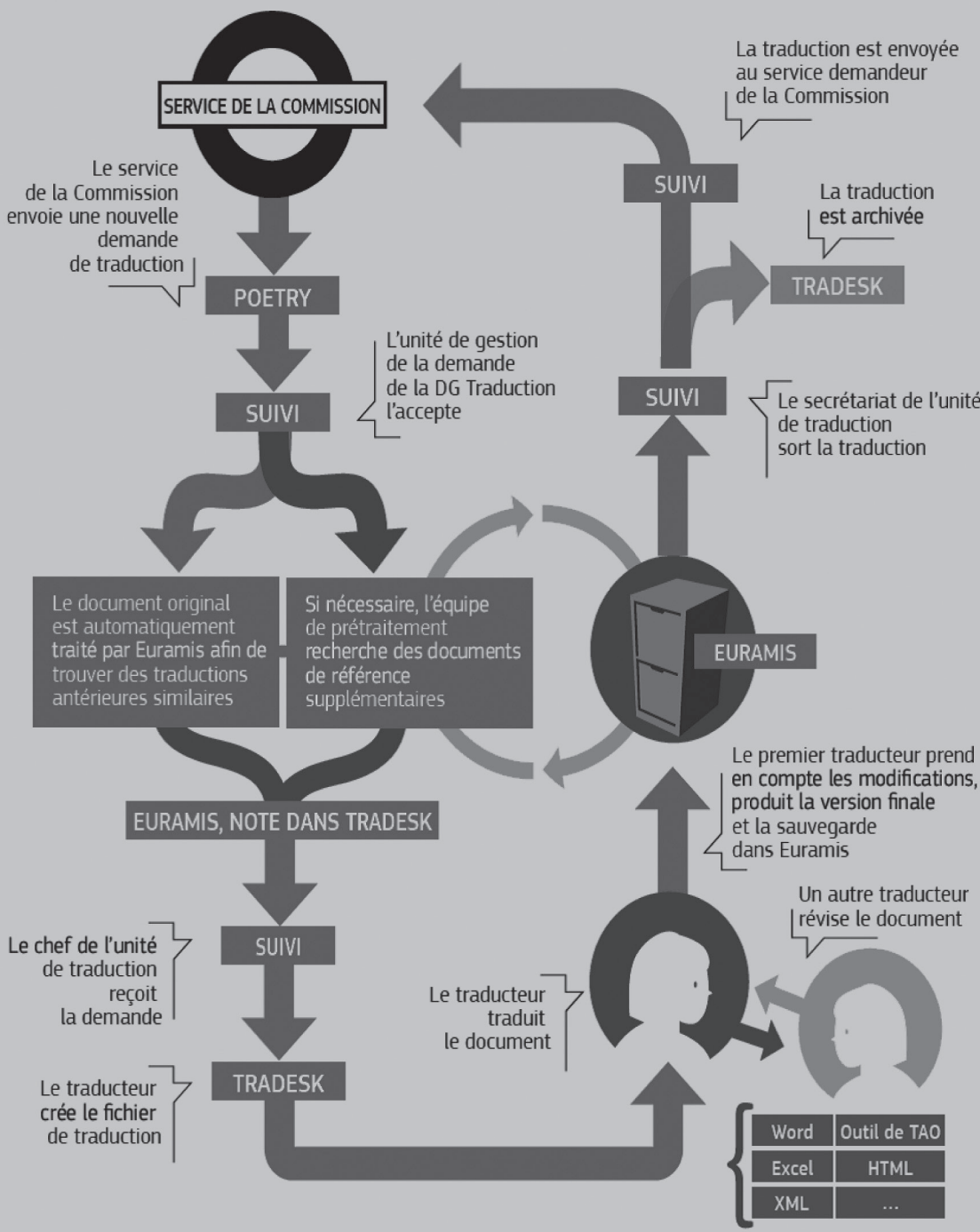

Figure 1. Cycle de la traduction à la Commission européenne (Commission européenne 2013, 5) 
En ce qui concerne les outils terminologiques, IATE (initialement appelée Inter-Agency Terminology Exchange et aujourd'hui connue comme InterActive Terminology for Europe), base de données terminologiques de l'ensemble des institutions européennes, occupe une place prépondérante. C'est la source centrale de la terminologie de l'UE qui réunit les anciennes bases terminologiques: EURODICAUTOM (Commission européenne), EUTERPE (Parlement européen) et TIS (Conseil européen).

En 1999, les institutions de l'Union européenne ont décidé d'élaborer une base de données entièrement neuve en fusionnant le contenu de toutes leurs bases de données terminologiques, afin de renforcer la coopération interinstitutionnelle tout en tirant partie des nouvelles technologies. En 2004, IATE a commencé à fonctionner pour l'usage interne des institutions européennes. En juin 2007, IATE a été mis à la disposition du grand public (http://iate.europa.eu.).

Aujourd'hui, IATE contient " plus de 8,7 millions de termes et un demi-million d'abréviations dans toutes les langues officielles de l'UE, auxquelles s'ajoute le latin » (Commission européenne 2013, 9).

Les résultats de la recherche mentionnent l'institution qui a créé l'entrée et le contexte d'utilisation du terme. Les entrées sont également accompagnées d'un code de fiabilité, un nombre d'étoiles (ou des chiffres dans la version anglaise) allant de 1 à 4 , où 4 étoiles signifient " très fiable » et 1 étoile " fiabilité non vérifiée " (Commission européenne 2013, 9).

EuroVoc est un thésaurus multilingue de l'Union européenne, disponible en 23 langues officielles (toutes les langues à l'exception de l'irlandais), ainsi qu'en serbe, albanais et macédonien. Il couvre 21 domaines suivants :

Vie politique

Relations internationales

Union européenne

Droit

Economie

Echanges économiques et commerciaux

Production, technologie et recherche

Questions sociales

Education et communication

Sciences

Entreprise et concurrence
Emploi et travail

Transports

Environnement

Agriculture, sylviculture et pêche

Agro-alimentaire

Energie

Finances

Industrie

Géographie

Organisations internationales 
Lancé en 1984, il résulte de la coopération de l'Office des publications officielles et du Parlement européen. Initialement, il englobe sept langues : l'allemand, I'anglais, le danois, le français, l'italien, le grec et le néerlandais. Gautier (2006) souligne que « la langue utilisée pour sa conception est le français, les autres langues sont des traductions "(Gautier 25). Au fur et à mesure de l'élargissement de l'UE, le nombre de langues disponibles augmente. Plusieurs parlements nationaux des pays candidats s'intéressent également à EuroVoc, en vue d'une meilleure préparation de leur adhésion à l'UE.

« Eurovoc est géré depuis 1999 par deux comités : le comité de pilotage et le comité de maintenance» (Gautier 27). Le premier établit les stratégies de développement, tandis que le deuxième met à exécution les décisions du premier.

\section{Cadre linguistique de l'adhésion à l'UE}

Avant de devenir membre de l'UE, un pays doit obligatoirement aligner sa législation nationale à la législation européenne. Pour être en mesure de le faire, d'un côté, il est tout d'abord nécessaire de le traduire dans la langue officielle du pays candidat. Vu que, selon Ramljak $(2008,166)$, l'acquis a atteint 128181 pages en 2008 et que ce nombre ne cesse de croître, il est évident qu'il s'agit d'un travail d'une grande complexité. De l'autre côté, la législation nationale doit être traduite dans l'une des langues officielle de l'UE (en principe, l'anglais) pour que son niveau d'alignement à l'acquis puisse être évalué lors du screening. Il s'agit donc d'un double processus de traduction qui requiert « un travail de recherche et de création terminologique considérable » (Commission européenne 2010, 20). Comme nous l'avons vu dans une étude sur le vocabulaire du droit de l'UE (Vušović 2019), il s'est avéré très hétérogène. Le fait qu'il englobe un très large éventail de domaines différents complique davantage le travail des traducteurs.

L'un des moyens d'aide à la traduction, mis à la disposition des pays candidats, est la base de données CCVista (http://ccvista.taiex.be) :

CCVista réunit les traductions des textes juridiques de l'UE dans les langues des pays candidats à l'adhésion et donne la possibilité de télécharger les textes du droit européen, repartis en différentes catégories, selon les phases de la révision.

Selon Robertson (2009), le fait de devenir une langue officielle de l'UE entraîne des conséquences pour la langue en question :

Les textes de l'UE exigent des termes équivalents dans toutes les langues. S'ils n'existent pas dans la langue nationale, ils doivent être créés. [...] Beaucoup de nouveaux mots voient le jour, non seulement les noms des concepts juridiques de l'UE (acquis commu- 
nautaire, règlement), mais aussi des termes techniques et économiques [...] : une lourde tâche pour un nouvel Etat membre. Dans les premières années à la suite de l'adhésion, la terminologie est un problème, puis elle se stabilise et s'établit (Robertson 2009, en ligne : http://bcom.au.dk/fileadmin/www.asb.dk/isek/robertson. pdf, consulté le 20 mars 2015). ${ }^{1}$

Au sujet de la standardisation dans les actes législatifs de l’Union européenne, Berteloot (2008) note que :

Le problème affecte différemment les langues de nouveaux États membres pour lesquels la traduction en bloc de l'acquis communautaire est à la fois un avantage et un inconvénient. La concomitance de la traduction de nombreux actes permet - avec les moyens technologiques appropriés - d'assurer une harmonisation terminologique. Mais la nécessité simultanée d'appréhender de nouveaux concepts n'ayant pas forcément de correspondance dans le système national et de créer un vocabulaire adéquat dans un laps de temps qui ne laisse pas le temps de la réflexion ne garantit pas non plus une qualité terminologique optimale des actes dans de nouvelles langues.

Il faut en outre reconnaître que certains concepts doivent se stabiliser et que leur expression peut varier aux premiers temps de leur introduction (2008, 18-19).

Comme le souligne Šarčević (2015):

La tâche délicate de créer des termes pour traduire la législation de l'UE est particulièrement aiguë dans les pays candidats où l'une des conditions tacites d'adhésion est la traduction de l'ensemble de l'acquis, une entreprise herculéenne exigeant la création de l'ensemble du vocabulaire du droit de l'UE dans la langue nationale dans une période relativement courte [...] II en résulte souvent une approche ad hoc à la formation de la terminologie [...] sans une stratégie globale ${ }^{2}(2015,183)$.

\footnotetext{
${ }^{1}$ « EU texts require equivalent terms in every language. If they do not exist in national language, they must be created. [...] Many new words are created, not only the names of EU legal concepts (acquis communautaire, regulation), but also technical and economic terms [...]: an onerous task for a new member state. For an initial period of years after accession terminology is a problem; then it settles down and becomes established. " (traduit par mes soins)

${ }^{2}$ " The sensitive task of creating terms to translate EU legislation is particulary acute in the candidate coutries where one of the tacit conditions for accession is the translation of entire acquis, a Herculean undertaking requiring the creation of the entire vocabulary of EU law in the national
} 
Outre les difficultés qui apparaissent au niveau terminologique, le volet syntaxique est également à mentionner, vu que certaines règles de rédaction de textes législatifs de l'UE y touchent. En effet, selon les Règles de technique législative à l'usage des services de la Commission (1985), il est stipulé que :

Pour des raisons d'ordre pratique, et notamment pour que les renvois puissent être identiques dans toutes les langues, la division des textes en phrases (une « phrase » étant la partie de texte comprise entre deux points) doit être la même dans toutes les versions. Lorsque ce principe soulève des difficultés dans une langue, on utilisera le point-virgule pour les coupures nécessaires $(1985,67)$.

Cette identité de paragraphes et de division en phrases dans toutes les versions linguistiques est censée faciliter les modifications ultérieures des actes en question. Toutefois, il n'est certainement pas simple de le mettre en pratique dans les 24 langues officielles et notamment dans les langues des pays en voie d'adhésion, toujours inexpérimentées en matière de la technique législative européenne. En examinant s'il est vraiment possible d'établir « une syntaxe européenne pour tout le monde ${ }^{3}$, Pym (2000) indique que ce principe "peut avoir certains effets négatifs au niveau de la lisibilité finale " vu qu'il néglige le fait que « les différentes langues ont des normes différentes pour les structures macro-textuelles $»^{4}(2000,3)$.

\section{Préparatifs dans les pays du BCMS}

Dans les lignes qui suivent, nous allons passer en revue la situation linguistique, à savoir les préparatifs et la mise en place de divers outils dans les pays de l'ancien serbo-croate : la Bosnie, la Croatie, le Monténégro et la Serbie. Vu que la Croatie est devenue le $28^{\mathrm{e}}$ Etat membre de l'UE le $1^{\text {er }}$ juillet 2013 et que, de ce fait, elle dispose des mécanismes les plus déployés en la matière, il convient de commencer par l'expérience croate.

\subsection{Croatie}

Selon l'article 69 de l'Accord de stabilisation et d'association entre les Communautés européennes et leurs États membres d'une part, et la République de Croatie, d'autre part, signé à Luxembourg en 2001 :

\footnotetext{
language over a relatively short period of time. [...] This often results in an ad hoc approach to terminology formation [...] without an overall strategy. » (traduit par mes soins)

${ }^{3}$ " European Syntax for All » (traduit par mes soins)

${ }^{4}$ « [...] it can have certain negative effects on the level of final readability. [...] different languages have different norms for macrotextual structures. " (traduit par mes soins)
} 
1. Les parties reconnaissent l'importance du rapprochement de la législation existante de la Croatie avec celle de la Communauté. La Croatie veille à ce que sa législation actuelle et future soit rendue progressivement compatible avec l'acquis communautaire.

2. Ce rapprochement débutera à la date de signature de l'accord et s'étendra progressivement à tous les éléments de l'acquis communautaire $[. .$.

Le processus de traduction s'est organisé autour de deux axes : d'un côté, il a fallu traduire l'acquis en croate et de l'autre côté, pour les besoins des négociations d'adhésion, il a fallu traduire les actes juridiques croates en l'une des langues officielles de l'UE, en l'occurrence, l'anglais. En vue de mener à bien ce processus, plusieurs outils d'aide à la traduction ont été élaborés :

- la version croate de l'EuroVoc, thésaurus multilingue de l'Union européenne ;

- Glossaire de l'ASA : anglais-croate, croate-anglais (http://www.mvep.hr ) ;

- Manuel de traduction des actes juridiques de l'UE (http://www.mvep. hr) ;

- Glossaire de services bancaires, assurances et autres services financiers (anglais-croate) (http://www.mvep.hr) ;

- Manuel de traduction des actes juridiques de la République de Croatie en anglais (http://www.mvep.hr) ;

- Dictionnaire quadrilingue du droit de l'UE (anglais-croate-français-allemand).

Comme nous pouvons le voir, les outils destinés à faciliter le travail des traducteurs consistent en dictionnaires, thésaurus, glossaires ainsi qu'en deux manuels de traduction, tous focalisés sur l'anglais, à l'exception du Dictionnaire quadrilingue, qui englobe également le français et l'allemand.

Dansla préface du Dictionnaire quadrilingue du droit de l'UE (Četverojezični rječnik prava Europske unije), Bratanić (2006) indique qu'il contient environ 5000 termes et qu'il s'appuie sur la version tchèque du même dictionnaire, où le tchèque a été remplacé par le croate. Elle souligne également que le choix de l'anglais comme point de départ pour la traduction n'est pas toujours bon, vu que la plupart des textes constituant le corpus pour l'élaboration du dictionnaire ont été initialement créés en français. Ramljak $(2008,168)$ met en évidence le même problème en rappelant que l'anglais n'est devenu la langue officielle de l'UE qu'en 1973 (lors de l'adhésion de l'Irlande et du Royaume-Uni) et que, jusqu'aux années 1990, les versions françaises ont été celles d'origine de $80 \%$ des actes juridiques de l'UE. De ce fait, afin d'assurer la qualité de traduction, il est souhaitable de recourir à la comparaison de plusieurs versions linguistiques. 
Dans l'introduction du Manuel de traduction des actes juridiques de l'UE (Novak 2002), Šarčević donne quelques conseils relatifs à la traduction de l'acquis. Elle note que le traducteur ne doit pas nécessairement être un spécialiste du droit européen, cependant, les connaissances de base sur l'UE, ses institutions et les textes juridiques européens, sont fortement recommandées. II est également conseillé de respecter strictement les formes standardisées des actes de l'UE, fournies par le Formulaire des actes.

Bratanić et Lončar (2015) rendent compte de l'incohérence terminologique dans les textes juridiques et essayent d'en détecter les causes. Elles expliquent que l'un des problèmes réside dans l'utilisation de plusieurs termes pour un seul concept dans le texte d'origine. Malgré les consignes précises selon lesquelles " il faut utiliser les mêmes termes pour exprimer les mêmes concepts " (Guide pratique commun, 6.2.) :

Le législateur de l'UE utilise parfois des synonymes même à l'intérieur d'un même texte. L'utilisation de plusieurs termes pour désigner le même concept est trompeuse, car elle suggère que les concepts sont également différents. A titre d'exemple, dans les versions consolidées du traité sur l'Union européenne (TUE) et du traité sur le fonctionnement de l'Union européenne (TFUE), le terme third country apparait 18 fois et le terme third State 4 fois ${ }^{5}$ (Bratanić et Lončar 2015, 213).

Elles notent que, dans la traduction croate, treća zemlja (third country) apparaît 21 fois et treća država (third State) une seule fois.

Pour Ostroški Anić (2009), l'harmonisation et la standardisation terminologiques sont d'une grande importance pour une traduction de qualité. De ce fait, elle note que les difficultés terminologiques auxquelles se heurtent les traducteurs peuvent être dues à l'inexistence des termes normalisés. En analysant les traductions croates de la version anglaise d'un règlement de l'UE relatif au domaine du transport aérien, elle note que le terme anglais airframe (en français cellule d'aéronef selon IATE $\left.{ }^{6}\right)$, apparaissant 10 fois, est rendu en croate par : konstrukcija zrakoplova (5 fois), zmaj zrakoplova (2 fois) et konstrukcija (zmaj) zrakoplova (3 fois).

Elle souligne que le traducteur est souvent tenté d'utiliser les synonymes pour résoudre le problème de la répétition de mêmes termes. "Toutefois, les

\footnotetext{
${ }^{5}$ « [...] the EU legislator sometimes uses synonyms even in the same text. Using more than one term to designate the same concept is misleading because it suggests that the underlying concepts are also different. For example, in the consolidates versions of the Treaty on European Union (TEU) and Treaty on the Functioning of the European Union (TFEU), the term third country occurs 18 times and third State four times. » (traduit par mes soins)

${ }^{6} \mathrm{http} / / /$ iate.europa.eu
} 
textes spécialisés et notamment les textes juridiques, ne sont pas destinés à l'expression de la créativité et ni à l'enrichissement du lexique " ${ }^{7}$ (Ostroški Anić $2009,45)$, de ce fait, il ne faut pas essayer de les rendre plus attrayants.

A ce propos, il faut mentionner le projet de normalisation de la terminologie croate et la création de la base terminologique Struna (strukovno nazivlje). Le projet a été entamé en 2008 sous la direction de l'Institut de langue et de linguistique croates (Institut za hrvatski jezik i jezikoslovlje). Selon Brač et Lončar (2012), les principes initiaux du travail terminologique au sein de ce projet ont été les suivants :

1) La terminologie fait partie de la langue croate standard.

2) Les experts de domaines particuliers ainsi que les linguistes devraient être inclus à la création de la terminologie.

3) Les linguistes devraient participer à la création terminologique dans tous les domaines et les experts de domaines particuliers devraient le faire pour leurs domaines respectifs.

4) La terminologie des domaines différents devrait être harmonisée.

5) La synonymie est indésirable. Les relations entre les synonymes devraient être précisées (terme préféré, terme admis, terme déconseillé, terme obsolète, terme familier).

6) En vue de la création du système terminologique, il est nécessaire de définir tous les termes ${ }^{8}(2012,262)$.

L'approche prescriptive adoptée par les protagonistes de ce projet nous rappelle le travail d'Eugen Wüster, que nous avons abordé dans une étude sur les approches théoriques de la terminologie (Vušović 2014). Bratanić et Ostroški Anić (2013) confirment que ces postulats ont été effectivement au cœur de la démarche : "le travail terminologique effectué dans le cadre de e-Struna est largement organisé selon les principes de la Théorie générale de la terminologie $(\text { TGT })^{9} »(2013,677)$.

\footnotetext{
7 « Međutim, stručni, a pogotovo pravni tekstovi, nisu namijenjeni izražavanju kreativnosti i bogaćenju leksika. » (traduit par mes soins)

${ }^{8} 1$. Terminology is part of the Croatian standard language.

2. Both subject field experts and linguists should be involved in the creation of Terminology.

3. Linguists should participate in creating terminology from all fields, and subject field experts should participate in the creation of terminology for their respective fields.

4. Terminology from different fields should be mutually harmonized.

5. Synonymy is undesirable, and relationships should be established among synonyms (preferred term, admitted term, deprecated term, obsolete term, colloquial term).

6. In order to create a terminology system, it is necessary to define all terms. (traduit par mes soins) ${ }^{9}$ « the terminological work carried out in e-Struna is largely organized according to the principles
} 
L'intention initiale était de créer une base de données terminologiques monolingue en vue de standardiser la terminologie croate. Néanmoins, « il a finalement été décidé que la base de données devrait être multilingue, avec les définitions uniquement en croate et les termes équivalents dans au moins une langue étrangère (anglais), ou de préférence dans plusieurs autres langues ${ }^{10}$ ॥ (2012, 267-268).

Un travail collaboratif entre linguistes, terminologues et experts de différents domaines spécialisés a abouti à une base de données qui contient actuellement environ 32000 termes (Brač et Lončar 2012). Selon le site officiel de Struna , 22 domaines suivants sont disponibles au public : anatomie et physiologie, anthropologie, archéologie, construction navale, technologie du bois, pharmacologie, phytomédecine, physique, construction, hydraulique et pneumatique, cartographie et géoinformatique, chimie, terminologie chimique et de laboratoire, corrosion et protection des matériaux, mathématiques, art dentaire, éléments de machine, aviation et terminologie militaire. Le traitement du terme est en cours dans les domaines de l'archéologie ancienne, de la criminalistique, de la génétique, de l'architecture classique, de la bibliothéconomie, de l'ophtalmologie, de la comptabilité et de la musicologie. ${ }^{11}$

\subsection{Bosnie-Herzégovine, Monténégro, Serbie}

A notre connaissance, en Bosnie-Herzégovine, au Monténégro et en Serbie, il n'y a pas eu de projets de normalisation terminologique, à l'instar de Struna croate. En vue de mener à bien le processus de traduction dans l'optique de l'adhésion à l'UE, les offices rattachés aux ministères de l'intégration européenne ont mis en place des outils suivants destinés à faciliter le travail des traducteurs :

En Bosnie-Herzégovine :

- BiHterm, base terminologique multilingue, contenant en majorité les équivalents anglais, et parfois incluant d'autres langues officielles de I'UE ;

- Glossaire de l'ASA (anglais - bosniaque/croate/serbe) ;

- Dictionnaire terminologique de l'intégration européenne (anglais bosniaque/croate/serbe) ;

- Manuel de traduction des actes juridiques de l'UE.

of the General Theory of Terminology (GTT). " (traduit par mes soins)

${ }^{10}$ " it was eventually decided that the database should be multilingual, with definitions only in Croatian and term equivalents in at least one foreign language (English), or preferably several other languages. " (traduit par mes soins)

${ }^{11} \mathrm{http}: / /$ struna.ihjj.hr/ , consulté le 13 avril 2019 
Au Monténégro :

- Monterm, base terminologique monténégrine, contenant 7143 termes en monténégrin et en anglais ${ }^{12}$;

- Montekorpus, corpus parallèle bilingue (anglais-monténégrin) recueillant des textes juridiques traduits ;

- Manuel de traduction des actes juridiques.

En Serbie :

- Evronim, base terminologique multilingue ${ }^{13}$;

- Evroteka, corpus parallèle bilingue (anglais-serbe) recueillant des textes juridiques traduits ;

- Manuel de traduction des actes juridiques de l'UE.

Comme nous pouvons le voir, ces outils comprennent les dictionnaires, glossaires, bases terminologiques et corpus parallèles. II est à constater qu'ils sont majoritairement orientés vers l'anglais, vu que c'est la langue depuis et vers laquelle on traduit le plus.

Les quatre manuels (bosniaque, croate, monténégrin et serbe) prennent des formes semblables. Vu que l'anglais est le plus souvent la langue à partir de laquelle on traduit, ils reproduisent la forme anglaise de différents types d'actes de l'UE (règlements, décisions, directives, recommandations, résolutions, conclusions, déclarations, accords internes et accords internationaux) et en proposent la forme BCMS. Les formes d'actes, disponibles dans plusieurs langues officielles de l'UE, sont issues du Manual of precedents for acts established within the Council of the European Union (Formulaire des actes établis dans le cadre du Conseil de l'Union européenne, 2002).

Outre les formes d'actes, les manuels comportent différentes consignes portant sur la structure des actes (titre, préambule, dispositif etc.), ainsi que les listes de pays membres, langues officielles, monnaies, sigles etc. Pour ce qui est des lignes directrices d'ordre linguistique, elles concernent essentiellement l'orthographe et la grammaire: majuscules/minuscules, nombres, emploi des temps verbaux et du passif. Cette fois-ci, l'anglais est de nouveau pris comme langue de départ.

\section{Conclusion}

Au fur et à mesure de l'élargissement de la construction européenne et de l'augmentation du nombre des langues officielles, la complexité de l'activité traduisante au sein de l'UE a été considérablement amplifiée.

\footnotetext{
${ }_{12}$ Disponible sur : http://www.eiprevod.gov.me/

${ }^{13}$ Disponible sur http://prevodjenje.seio.gov.rs/evronim/
} 
Les années initiales sont caractérisées par les effectifs de traducteurs et de réviseurs assez modestes. Les outils technologiques dont ils se servent quotidiennement sont peu développés. Au fil du temps, l'automatisation du travail donne naissance aux premières bases terminologiques. L'essor de l'informatique introduit la mise en place de différents mémoires de traduction, moteurs de recherche et logiciels. Le nombre de langues officielles grandit considérablement et le flux du travail ne fait qu'augmenter.

Aujourd'hui, des effectifs considérables, une production qui ne cesse de croître, ainsi que les difficultés auxquelles se heurtent les traducteurs de l'UE dans leur travail quotidien, placent les services de traduction des institutions européennes parmi les plus impressionnants au monde.

Vu que l'adhésion d'un pays à l'UE sous-entend l'adoption de la législation européenne et de ce fait, la traduction de l'acquis dans la langue du pays candidat, une lourde tâche incombe aux traducteurs nationaux. Les défis auxquels ils doivent faire face sont nombreux : s'investir dans un travail complexe de création terminologique pour trouver des équivalents appropriés, souvent pour les concepts encore flous et inconnus, respecter strictement les formes standardisées des actes juridiques de l'UE, se conformer aux exigences d'ordre syntaxique et textuel, identiques pour toutes les versions linguistiques, résoudre les problèmes de l'interférence avec l'anglais, qui est le plus souvent la langue à partir de laquelle on traduit etc.

En ce qui concerne les préparatifs que les pays du BCMS ont effectués afin de répondre à ces défis, l'on constate les démarches presque identiques qui comprennent l'élaboration de plusieurs dictionnaires, glossaires, corpus parallèles et manuels de traduction. La Croatie, dont la langue figure maintenant parmi les langues officielles de l'UE, a également procédé à la normalisation terminologique dans le cadre de projet Struna.

Comme nous avons pu le voir, il s'agit des outils majoritairement orientés vers l'anglais, ce qui constitue une démarche jusque-là logique. Or, après le Brexit, le statut de l'anglais sera-t-il remis en question ? Perdra-t-il le statut de langue officielle (I'Irlande ayant choisi l'irlandais et Malte le maltais) ? Resterat-il la langue du travail, à côté du français et de l'allemand ? Dans l'incertitude qui plane autour de ces questions, une chose semble être certaine : le nombre de locuteurs natifs travaillant dans les institutions européennes baissera inévitablement. A quel anglais hybride aura-t-on affaire ? Pour toutes ces raisons, le fait de se limiter sur l'anglais est de toute évidence une pratique à remettre en question.

Pour ce qui est des facteurs internes au sein des pays candidats, la nécessite de l'aménagement terminologique, à l'instar de Struna croate, devient évidente. L'importance des projets de normalisation et d'harmonisation terminologique, ainsi que le besoin d'un travail collaboratif entre linguistes et experts de différents domaines spécialisés est fortement recommandable. 
La totalité des travaux qui constituent le cadre linguistique de l'adhésion est une entreprise " herculéenne " (Šarčević 2015). En outre, il faut penser à l'avenir du BCMS dans l'Union européenne, car l'adhésion n'est pas seulement d'ordre politique, institutionnel et juridique, mais également de nature linguistique et culturelle. Afin de consolider la future place de notre langue au sein du riche éventail des langues officielles de l'UE, il importe de penser dès maintenant à ce travail interne qui nous revient.

\section{Références bibliographiques}

Accord de stabilisation et d'association entre les Communautés européennes et leurs États membres d'une part, et la République de Croatie, d'autre part [JO L 026 du 28/01/2005]

Berteloot, Pascale. " La standardisation dans les actes législatifs de l'Union européenne et les bases de terminologie. "Normalisation, harmonisation et planification linguistique. Ed. Elena Chiocchetti et Leonhard Voltmer. Bolzano: Publications EURAC Research. 2008. 11-18.

Brač, Ivana, and Maja Lončar. « Terminology planning for the croatian national terminology database STRUNA. " Proceedings of the 10th Terminology and Knowledge Engineering Conference (TKE 2012). Madrid, 2012.

Bratanić, Maja, ed. Četverojezični rječnik prava Europske unije, Zagreb: Hrvatska informacijsko-dokumentacijska referalna agencija, 2003.

Bratanić, Maja, and Ana Ostroški Anić. « The Croatian national termbank STRUNA: A new platform for terminological work. " Collegium antropologicum 37.3 (2013): 677-683.

Bratanić, Maja, and Maja Lončar. " The Myth of EU Terminology Harmonization on National and EU Level. " Language and Culture in EU Law: Multidisciplinary Perspectives (2015): 207.

Commission européenne. Règles de technique législative à l'usage des services de la Commission. Bruxelles, 1985.

Commission européenne. La traduction à la Commission: 1958-2010. Luxembourg : Office des publications officielles, 2009.

Commission européenne. Histoire de la traduction à la Commission européenne. Luxembourg : Office des publications officielles, 2010.

Commission européenne. Traduction et multilinguisme. Luxembourg : Office des publications officielles, 2012.

Commission européenne. Outils d'aide à la traduction et cycle de travail. Luxembourg : Office des publications officielles, 2013.

De Vicente, Francisco. European Union and Translation. Bruxelles: Directorate General for Translation, 2011. En ligne: http://ec.europa.eu/dgs/translation/workwithus/candidatecountries/documents/european union translation_en.pdf, consulté le 15 mars 2015. 
Gautier, Isabelle. « Eurovoc, thésaurus multilingue: Maintenance et aspects pratiques. Exemple à la bibliothèque du parlement européen. " Cahiers de la documentation 60.1 (2006): 25-34.

Guide pratique commun pour la rédaction des textes législatifs communautaires à l'intention des personnes qui contribuent à la rédaction des textes législatifs. Luxembourg : Office des publications, 2003.

Mauro, Cristina et Francesca Ruggieri, eds. Droit pénal, langue et Union européenne: réflexions autour du procès pénal. Bruxelles : Bruylant, 2012.

Novak, Jasminka, ed. Priručnik za prevođenje pravnih akata Europske unije. Zagreb: Ministarstvo za europske integracije Republike Hrvatske, 2002.

Ostroški Anić, Ana. „Utjecaj prevođenja zakonodavstva EU-a na normiranje hrvatske zrakoplovne terminologije." Lingvistika javne komunikacije: Translatološki, terminološki, međukulturni i problemi jezika struke. Ed. Omazić, Marija, Vladimir Karabalić i Mario Brdar. Zagreb - Osijek : Hrvatsko društvo za primijenjenu lingvistiku - Filozofski fakultet Sveučilišta Josipa Jurja Strossmayera, 2009. 41-48.

Pellerin-Rugliano, Caroline. "Traducteur juriste ou juriste traducteur? Quelques aspects de la traduction à la Cour de justice de I'Union européenne. ॥ La traduction juridique : Points de vue didactiques et linguistiques. Ed. Meunier, Mariette, Marion Charret-Del Bove et Eliane Damette. Lyon : Université Jean Moulin Lyon 3, Publications du Centre d'Etudes Linguistiques. 2013. 258-280.

Pym, Anthony. "The European Union and its future languages. Questions for language policies and translation theories. "Across languages and cultures 1.1 (2000): 1-17.

Ramljak, Snježana. « “Jezično” pristupanje Hrvatske Europskoj Uniji: prevođenje pravne stečevine i europsko nazivlje. » Politička misao 45.01 (2008): 159177.

Robertson, Colin. " LSP and EU legal language. " Reconceptualizing LSP. Online proceedings of the XVII European LSP Symposium. Ed. Heine, Carmen and Jan Engberg, 2009. En ligne: http://bcom.au.dk/fileadmin/www.asb.dk/ isek/robertson.pdf, consulté le 20 mars 2015.

Secrétariat général du Conseil. Formulaire des actes établis dans le cadre $d u$ Conseil de l'Union européenne, Luxembourg : Office des publications officielles, 2002.

Šarčević, Susan. Basic Principles of Term Formation in the Multilingual and Multicultural Context of EU Law. Language and culture in EU law: multidisciplinary perspectives. Ed. Šarčević, Susan. Farnham, Surrey, England ; Burlington, VT : Ashgate. 2015. 183-206.

Truchot, Claude, éd. Le plurilinguisme européen: Théories et pratiques en politique linguistique. Champion, 1994. 
Vušović, Olivera. "Approches théoriques de la terminologie et nature de termes : quelques considérations. ॥ Studii de Ştiintă şi Cultură 10.4 (2014) : 83-90.

Vušović, Olivera. "Vocabulaire du droit de l'Union européenne et l'adhésion du Monténégro: de l'analyse lexicométrique aux interprétations linguistiques. " Revue roumaine de linguistique. 64.1 (2019) : 83-98.

\section{Sitographie}

IATE : http://iate.europa.eu

CCVista Translation Database : http://ccvista.taiex.be

Ministère des affaires étrangères et européennes (Croatie) : http://www.mvep.hr Monterm : http://www.eiprevod.gov.me

Evronim : http://prevodjenje.seio.gov.rs

Struna : http://struna.ihjj.hr/

\section{TRANSLATION TOOLS FOR EU LEGISLATION: EUROPEAN PRACTICES AND THE CASE OF BCMS}

The aim of this paper is to analyse the issue of memory through tools for storing linguistic data (terminological databases, parallel corpora, thesauri, glossaries and dictionaries) deployed in the context of the translation of EU legislation. On the one hand, we present an overview of the tools used within the $\mathrm{EU}$, distinguished by a complex and highly specific linguistic regime, currently including 24 official languages. On the other hand, we review the preparations and implementation of various tools in the former Serbo-Croatian countries, namely the language framework of their accession to the EU. Within the BCMS languages, a parallel has to be drawn between Croatia, which has been a member of the EU since 2013, with the most developed mechanisms in this area, of the one part, and Montenegro, Serbia and Bosnia and Herzegovina, of the other part.

Keywords: European Union, translation, tools, databases, accession to the EU, BCMS. 